\title{
Profiling of microRNAs and mRNAs in marine mussel Mytilus galloprovincialis
}

\author{
Deliang $\mathrm{Yu}^{\mathrm{a}}$, Huifeng $\mathrm{Wu}^{\mathrm{b}}$, Xiao Peng ${ }^{\mathrm{a}, *}$, Chenglong $\mathrm{Ji}^{\mathrm{b}}$, Xiaoying Zhang ${ }^{\mathrm{c}}$, Jun Song ${ }^{\mathrm{a}}$, Junle $\mathrm{Qu}^{\mathrm{a}}$ \\ ${ }^{\text {a }}$ Key Laboratory of Optoelectronic Devices and Systems of Ministry of Education and Guangdong Province, College of Physics and Optoelectronic Engineering, Shenzhen \\ University, Shenzhen 518060, PR China \\ ${ }^{\mathrm{b}}$ Key Laboratory of Coastal Zone Environmental Processes and Ecological Remediation, Yantai Institute of Coastal Zone Research (YIC), Chinese Academy of Sciences \\ (CAS), Shandong Provincial Key Laboratory of Coastal Zone Environmental Processes, YICCAS, Yantai 264003, PR China \\ ${ }^{\mathrm{c}}$ AstraZeneca-Shenzhen University Joint Institute of Nephrology, Department of Physiology, Shenzhen University Health Science Center, Shenzhen University, 518060, PR \\ China
}

A R T I C L E I N F O

\section{Keywords:}

Biological processes

MicroRNAs

mRNA

Mytilus galloprovincialis

\begin{abstract}
A B S T R A C T
MicroRNAs (miRNAs) are a class of noncoding RNA molecules containing 18-24 nucleotides, and those with conserved structures are able to regulate the expression of eukaryotic genes by inhibition or enhancement of mRNA translation. However, miRNAs of the blue mussel, Mytilus galloprovincialis have not been reported. $M$. galloprovincialis is a primary species distributed along coastal zones worldwide. To reveal the repertoire of miRNAs in M. galloprovincialis, we constructed small RNA libraries prepared from three different mussels, which were then sequenced by Solexa deep sequencing technology. A total of 32,836,817, 33,359,113 and 33,093,562 clean reads from the tissues of the three $M$. galloprovincialis were obtained. Based on sequence similarities and hairpin structure predictions, 137 M. galloprovincialis miRNAs (mg-miRNA) were identified. Among the mgmiRNAs, 104 were conserved across species, whereas 33 might be novel and specific for M. galloprovincialis. Some of the mg-miRNAs, such as let-7 and the miR-100 family are playing key roles in many metabolic pathways and are worthy of further study. By performing a whole genome-scale characterization of mg-miRNAs and proposing their potential functions, these results provide a foundation for understanding the biological processes of the blue mussel, M. galloprovincialis.
\end{abstract}

\section{Introduction}

The blue mussel (Mytilus galloprovincialis) is one of the main species cultured for seafood along the coastal areas of China and many coastal zones around the world. Mussels are also widely used marine organisms for environmental monitoring and assessment programs because of their sessile filter-feeding lifestyle, and high accumulation of pollutants from marine environments (Alimba and Faggio, 2019; Faggio et al., 2018; Stara et al., 2020; Torre et al., 2013). Marine mussels are well known because they are listed in the "Mussel Watch" program, which is a global program aimed at monitoring marine environments (Farrington et al., 2016). To understand the environmental adaptation mechanisms of $M$. galloprovincialis, researchers have studied multiple genes and proteins (Capillo et al., 2018; Faggio et al., 2016; Ji et al., 2013; Yu et al., 2016). However, the functions of most molecules remain unclear.

MicroRNAs (miRNAs) are a class of small noncoding endogenous RNA molecules containing 18-24 nucleotides that function as negative regulators of target genes. miRNAs regulate gene expression by directing their target mRNAs for degradation or translational repression (Ambros, 2004; Bartel, 2004). Increasing evidence have shown that miRNAs are primary regulators in cell differentiation and growth, mobility, apoptosis (programmed cell death) and immune responses (Aboobaker et al., 2005; Bueno and Malumbres, 2011). Thus far, $\sim 48,885$ mature miRNAs have been identified from 271 species (www. mirbase.org, release 22.0), with many others yet to be identified. In mollusks, only 245 miRNAs have been identified, from Haliotis rufescens, Lottia gigantean, and Melibe leonina.

MiRNAs have key roles in multiple biological processes in almost all species (Burgos-Aceves et al., 2018a). For many marine species, some novel miRNAs in marine medaka Oryzias melastigma target genes in a tissue-specific manner, such as neuron development and synaptic transmission in the brain, glucose and fat metabolism in the liver, and steroid genesis in the gonads. Such studies have led to further biomarker development for the assessment of environmental stresses and pollution in marine environments (Burgos-Aceves et al., 2018b, 2018c; Lai et al., 2015). In the marine oyster Crassostrea gigas, an invertebratespecific miRNA was identified to have a negative role in both the

\footnotetext{
* Corresponding author.

E-mail address: pengxiao_px@szu.edu.cn (X. Peng).
} 
synthesis and release of acetyl choline (ACh) and choline uptake in hemocytes during the early stages of pathogen infection (Chen et al., 2016). In another study, miRNA expression profiles in scallops (Chlamys farreri) were characterized in response to acute viral necrosis virus (AVNV) infection (Chen et al., 2014). The discovery of miRNAs in these studies has added a new dimension to our understanding of complex genetic regulatory networks in marine animals. However, the functions of miRNAs in marine mussels remain unclear.

Transcriptomics includes the analysis of a comprehensive set of transcripts expressed in cells under different conditions (e.g. a specific developmental stage or under stress), whereas RNA sequencing (de novo RNA-seq) technologies provide cost-effective high-throughput sequencing to advance genomic studies of non-model species. In many marine species, comprehensive genomic databases have been successfully constructed using RNA-seq, such as the owl limpet Lottia gigantea (Simakov et al., 2013), apple snail Pomacea canaliculata and pearl oyster Pinctada martensii (Sun et al., 2012; Jiao et al., 2014). However, miRNAs in Mytilus galloprovincialis (mg-miRNAs) have remained unclear. In the current study, we identified multiple mg-miRNAs and mRNAs in M. galloprovincialis and explored their potential functions by constructing small RNA libraries prepared with Solexa deep sequencing technology using the soft tissues of $M$. galloprovincialis. The results serve as a basis for further research on multiple biological processes in this organism, and establish a more comprehensive database of the miRNA and mRNA transcriptome of soft tissues in M. galloprovincialis.

\section{Methods}

\subsection{Experimental samples and RNA extraction}

Mussels (shell length: $3.5-5 \mathrm{~cm}, n=3$ ) were purchased at random from a fishery market in Yantai. The mussels were then transported to the laboratory and acclimatized in aerated normal filtered seawater (FSW) (salinity $31 \mathrm{psu}$, temperature $20{ }^{\circ} \mathrm{C}$ ) for 7 days. During the acclimation period, all mussels were cultured under a $12 \mathrm{~h}: 12 \mathrm{~h}$ light: dark photoperiod, and fed daily with Chlorella vulgaris at a ratio of $2 \%$ of tissue per dry weight. Soft tissues were peeled carefully from the shells and quickly dropped into liquid nitrogen. Then total RNA of soft tissues was extracted with TRIzol reagent (Invitrogen) according to the manufacturer's instructions. To identify the maximum number of miRNAs and mRNAs, the total RNAs from all of the tissue samples were pooled in equal fractions.

\section{2. miRNA and mRNA sequencing}

In this study, BGISEQ-500 deep sequencing technology (Huada Gene, Shenzhen, Guangdong, China) was applied to sequence short reads. Clean sequencing reads were obtained by removing reads that contained poly- $N$ with $5^{\prime}$ adapter contaminants, without $3^{\prime}$ adapters or the insert tag, containing poly A, T, G or C, low-quality reads, and reads $<18 \mathrm{nt}$. Following read clean-up, the high-quality reads were mapped to a reference sequence using Bowtie (Hoen et al., 2008), without mismatch to analyze their expression and distribution on the reference sequence. To remove tags originating from protein-coding genes, repeat sequences, rRNA, transfer RNA (tRNA), small nuclear RNA (snRNA), and small nucleolar RNAs (snoRNAs), sRNA tags were mapped to the RepeatMasker, Rfam database. The clean reads were compared with the miRNA precursor and/or mature miRNAs of all animals in miRBase (version 22.0) to identify the sequence and number of miRNA families (not species specific) in the samples. The characteristics of the hairpin structures of miRNA precursors were evaluated to predict novel miRNAs.

For mRNA sequencing, low-quality reads ( $>20 \%$ of the bases qualities being $<10$ ), reads with adaptors, and reads with unknown bases ( $\mathrm{N}$ bases $>5 \%$ ) were filtered to obtain the clean reads. These were then assembled into unigenes, followed by unigene functional annotation, simple sequence repeat (SSR) detection, and unigene expression level calculations. Finally, differential expressed genes (DEGs) were identified between samples and clustering analysis and functional annotations were performed. Raw reads were defined as reads containing low-quality, adaptor-polluted, and a high content of unknown base $(\mathrm{N})$ reads; such reads were also removed before downstream analyses. Internal software was used to filter reads, as follows: (1) remove reads with adaptors; (2) remove reads in which unknown bases (N) were $>5 \%$; and (3) remove low-quality reads (i.e., reads in which the percentage of bases with a quality $<10$ was $>20 \%$ in a read). After filtering, the remaining reads were called 'Clean Reads' and stored in FASTQ format.

In the de novo assembly, Trinity was used to perform a de novo assembly with clean reads (PCR duplication was removed to improve the efficiency) and then TGICL (TIGR Gene Indices clustering tools) was used to cluster transcripts to unigenes. Trinity contains three independent software modules: Inchworm, Chrysalis, and Butterfly. It partitions the sequence data into many individual de Bruijn graphs, each representing the transcriptional complexity at a given gene or locus, and then processes each graph independently to extract fulllength splicing isoforms and to tease apart transcripts derived from paralogous genes.

\section{3. miRNA and mRNA expression analysis}

To identify the miRNAs differentially expressed between three different mussels, expression data were $\log _{2}$-transformed and plotted on a scatter plot. Briefly, miRNA expression data from the three libraries were normalized to obtain the number of transcripts per million reads (TPM), using the normalization formula:

Normalized expression $=$ mapped readcount $/$ Total reads $* 1 \times 10^{6}$

TPM analysis step could eliminate the influence of sequencing discrepancies on the calculation of miRNA expression. The fold-change and $p$-values were calculated from the normalized expression and the $p$ values were then adjusted using the $q$-value. The $q$-value $<0.01$ and $\mid \log _{2}$ (foldchange) $\mid>1$ was set as the threshold for significantly different expression (default). Finally, the $\log _{2}$-ratio figure and scatter plot were generated. When the normalized expression of a miRNA was zero between three libraries, its expression value was adjusted to 0.01 (because 0 cannot be plotted on a log plot). If the normalized expression of a specific miRNA in three libraries was $<1$, further differential expression analyses were conducted without that miRNA.

\subsection{Transcriptome annotation}

Gene Ontology (GO; www.geneontology.org) is an international standard classification system for gene function. The distribution of those selected target genes (miRNA and mRNA) among biological pathways/functions in gene ontologies will be identified to character the biological differences between samples based on gene function. Using this method, candidate target genes were mapped to the GO terms (biological functions) in the database (www.geneontology.org). The number of genes in every term was calculated, and a hypergeometric test was performed to identify significantly enriched GO terms in the target gene candidate list from the background reference gene list.

The Kyoto Encyclopedia of Genes and Genomes (KEGG; www.kegg. jp) database is a public database containing biological pathway data, and is a resource for understanding high-level functions and processes active in a biological system (Kanehisa et al., 2008). In our work, KEGG pathway analysis helps us identify significantly enriched metabolic pathways or signal transduction pathways enriched in target gene 
candidates, compared with a reference gene background, using the hypergeometric test.

\subsection{Statistical analysis}

The R statistical software package ( $R$, Auckland, New Zealand) was used to analyze for the RNA-seq data. $\mathrm{R}$ was used for graphical representations, as well as to correct for multiple testing and $p$-value corrections. The data were expressed as the mean \pm standard error, with $p<0.05$ indicating a significant difference. Graphpad 5.0 (San Diego, CA, USA) was used to generate figures.

\section{Results}

\subsection{Overview of miRNA and mRNA-seq data}

To identify miRNAs that were differentially expressed in three mussels, three miRNA libraries were constructed by Solexa sequencing. The error rate of the sequencing data from the three libraries was $0.01 \%$, and Q30 was $>97.8 \%$, indicating that the sequencing data were of high quality and suitable for this study. A total of $32,836,817$, $33,359,113$, and $33,093,562$ reads were acquired from the M. galloprovincialis soft tissues of the three mussels. After discarding the sequences that were below the background, $76.56 \%, 81.71 \%$, and $68.08 \%$ of the miRNAs were mapped to the mussel (Bathymodiolus platifrons) genome, for which all genomic information has been reported and have a close genetic relationship with M. galloprovincialis (Sun et al., 2017). Subsequently, all identical reads were classified by group, providing $7,697,619,6,100,958$, and 10,564,320 unique sequences, respectively. The specific miRNA length ranges from the clean reads were subtracted from these sequences and the length distribution of miRNAs is shown in Fig. 3. Most of the miRNAs were 21-24 nucleotides in length. Therefore, the calculated gene expressions were directly used to compare the differences in gene expression between samples. A total of $32,836,817$, $33,359,113$, and $33,093,562$ miRNAs were obtained for each of the three samples. The remaining reads were further compared by using the software RepeatMasker and Rfam database, to remove possible mRNA, rRNA, tRNA, snRNA, snoRNA, and repeat sequences. However, because some sRNA tags might map to more than one category, the following priority rule was applied: rRNA etc. (Genbank $>$ Rfam) $>$ known miRNA $>$ repeat $>$ exon $>$ intron. All the clean reads were then divided into the following categories: exon_sense, exon_antisense, intron_sense, intron_antisense, miRNA, rRNA, repeat, scRNA, snRNA, snoRNA, srpRNA, tRNA, and unknown (sequences not mapped to any known reference databases). The composition of the RNA classes in each library is shown in Fig. 1. Among the clean reads, 25,139,198 (76.56\%), 27,258,155 reads (81.71\%) and 22,529,242 (68.08\%) were mapped to the mussel genome (Sun et al., 2017). Known miRNAs accounted for $77.61 \%, 77.61 \%$ and $77.78 \%$ of the total clean reads. The analyses of these three libraries confirmed that these miRNA sequences were enriched among the miRNA libraries.

As a result of mRNA sequencing, $19.85 \mathrm{~Gb}$ bases were generated in total on the BGISEQ-500 sequencing platform. Assembling all the samples and filtering the abundance resulted in 126,858 unigenes; the total length, average length, N50, and GC content of these unigenes were $139,638,363 \mathrm{bp}, 1100 \mathrm{bp}, 2142 \mathrm{bp}$, and $34.32 \%$ respectively. The unigenes were annotated by aligning them with seven functional databases, resulting in 55,037 (NR: 43.38\%), 13,094 (NT: 10.32\%), 38,282 (Swissprot: 30.18\%), 34,413 (KOG: 27.13\%), 40,065 (KEGG: 31.58\%), 8055 (GO: 6.35\%), and 46,648 (InterPro: 36.77\%) annotated unigenes (Fig. 2). For functional annotation results, 61,949 CDS (Coding sequences) were detected by Transdecoder, 9061 SSR were detected distributed on 7847 unigenes, and 7683 transcription factor (TF)-coding unigenes were predicted.

\subsection{Identification of miRNAs and potential novel miRNAs}

To identify known miRNAs in M. galloprovincialis, the data set was compared with miRNAs (miRNA precursors and mature miRNAs) in the miRBase (version 22.0). The three samples provided a total of

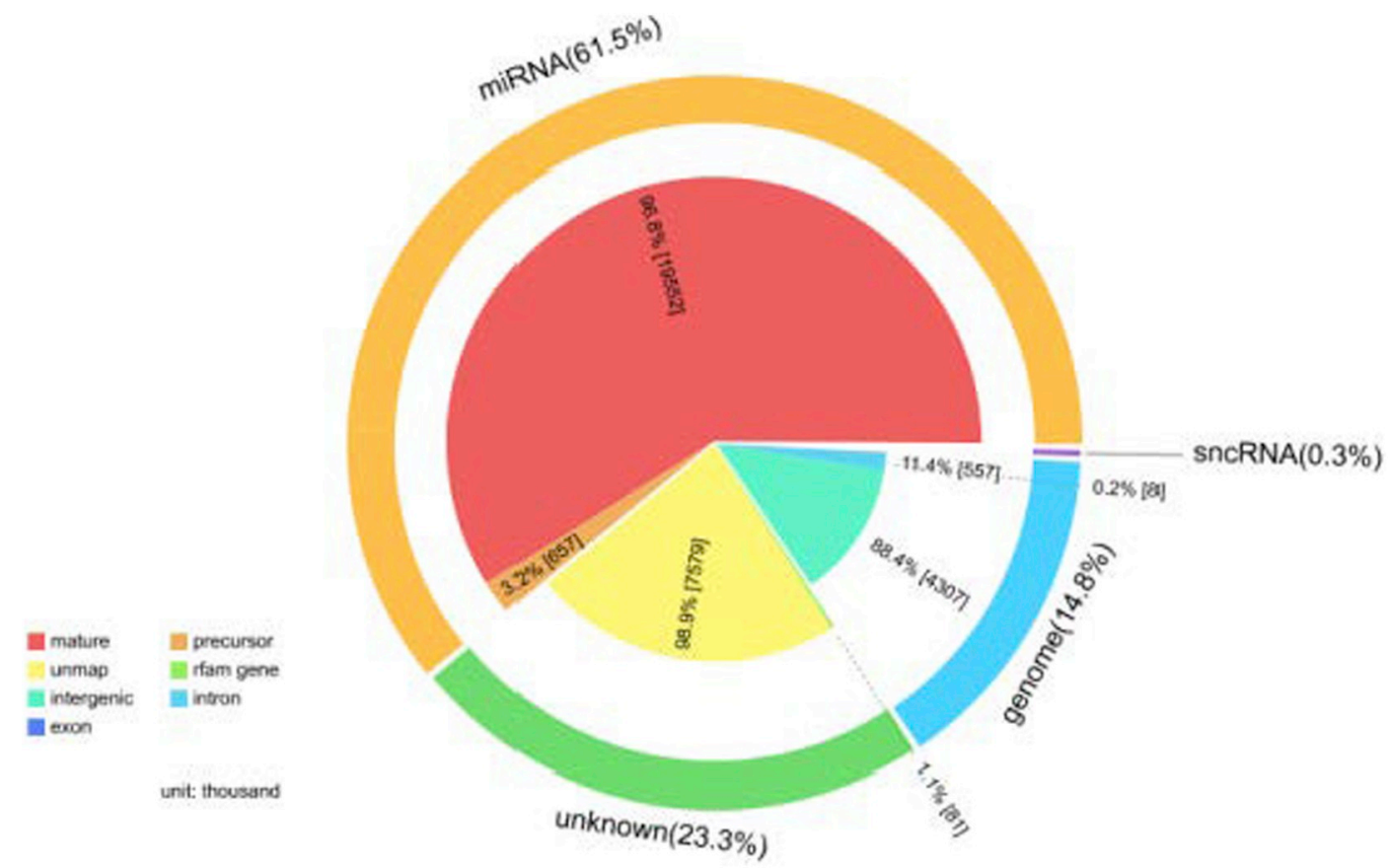

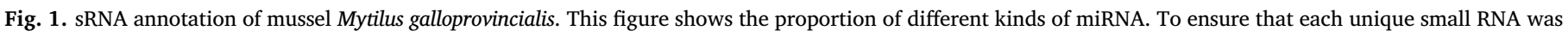
only mapped to one annotation, the following priority rule was applied: miRNA $>$ piRNA $>$ snoRNA $>$ Rfam $>$ other sRNA. 


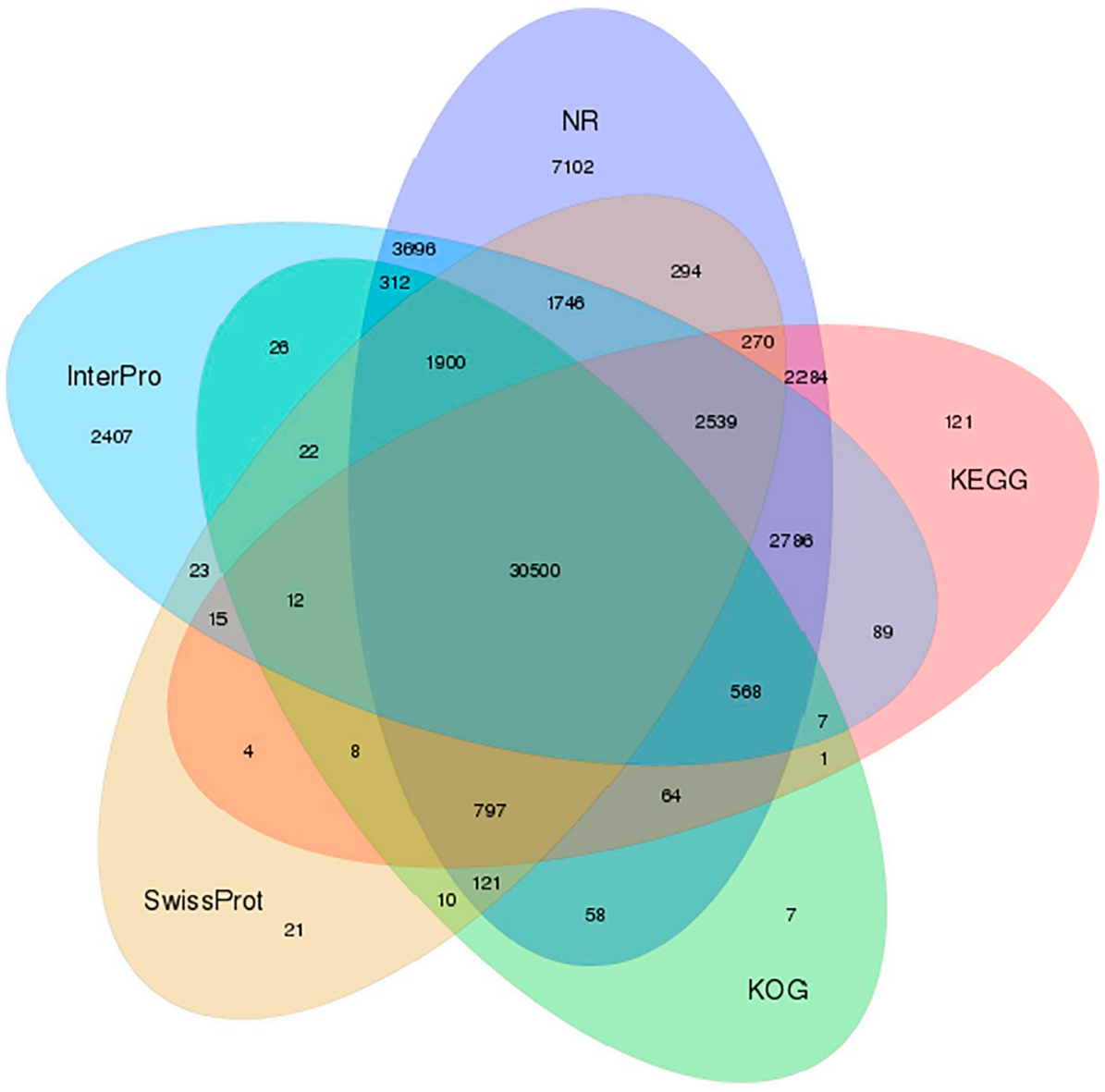

Fig. 2. Venn diagram between NT, NR, GO, KOG, KEGG, Swissprot and Interpro. These are functional databases (for more details, please find the official website below). Blastn, Blastx, or diamond were used to align unigenes to the NT, NR, KOG, KEGG and SwissProt databases for annotations, Blast2GO with NR annotation was used for GO annotations, and InterProScan 5 was used for the InterPro annotation.
$25,139,198,27,258,155$, and 22,529,242 unique sequences, respectively. Among these, 104 were mature miRNAs, as listed in Table 1.

A cDNA library of mg-miRNAs was sequenced using the BGISEQ500 sequencing platform. After removal of the low-quality and adaptor sequences, 27,479,838 reads representing 317,630 distinct sequences were obtained. Given that miRNAs with known functions are typically 18-24 bp in length, the unique size distribution patterns of the miRNAs were analyzed (Fig. 1). The results showed that the size of miRNAs was not evenly distributed, with the number of 22-bp sequences being significantly higher than the number of sequences with $<22$ or $>22 \mathrm{bp}$. The same result was observed for $34.48 \%$ of all sequences, which is consistent with the distribution of the most common sizes of miRNAs in other species (Ambros, 2001). Therefore, this analysis suggests that miRNAs in the mg-miRNA library are enriched as designed.

The presence of a hairpin RNA structure is a typical characteristic of miRNA precursor, which can be applied to predict novel miRNAs. Therefore, miRDeep2 miRNA prediction software was applied to identify potential novel miRNAs. Briefly, the precursor sequences were mapped to the mollusk genome by exploring their secondary structures, Dicer cleavage sites, and binding energies, leading to the identification of 33 potential novel miRNAs (Table 2).

\subsection{Transcriptome annotation analysis}

GO enrichment assessments were applied to analyze the candidate target genes for differentially expressed miRNAs to predict biological functions. Statistical analyses of the significantly enriched number of genes in each term are summarized in Fig. 4. KEGG pathway analyses showed that the candidate genes of miRNAs in the soft tissue of $M$. galloprovincialis were involved in 325 pathways. Moreover, the results indicated that several enriched terms were involved in biological processes, including cancer pathways, focal adhesion, PI3K-Akt signaling pathway, endocytosis, and neuroactive ligand-receptor interactions (Fig. 4).

\section{Discussion}

MiRNAs are important molecules in a series of mammalian biological processes (Bartel, 2018). An increasing number of miRNAs have been identified in various species, including marine animals (Chen et al., 2014; He et al., 2017; Huo et al., 2017). The blue mussel (M. galloprovincialis) is a popular sea food worldwide, produced in huge quantities, and is also used for monitoring the marine environment (Freitas et al., 2019; Pagano et al., 2016; Pagano et al., 2017). However, the functions of most molecules remain unclear, especially of noncoding RNA, for instance, miRNA. Lists of miRNAs have been reported for a few molluscan species (Chen et al., 2017; Martín-Gómez et al., 2014a; Zheng et al., 2016; Zhou et al., 2014) and miRNA families have been investigated in the limpet genome (Kenny et al., 2015). Given that a general overview of $M$. galloprovincialis miRNA was lacking, the current study used transcriptomic data sets available for $M$. galloprovincialis to identify and characterize core elements involved in the miRNA formation pathway in this mussel.

BGISEQ-500 deep sequencing technology is used to sequence shorter reads. The specified size of miRNA and mRNA molecules can be directly sequenced in the sample by this technology without any miRNA sequence information to provide a more comprehensive miRNA expression profile and expedite the discovery of new miRNAs (Huang 
Table 1

The information of mature miRNAs in the mussel Mytilus galloprovincialis.

\begin{tabular}{|c|c|c|c|c|}
\hline miRNA id & Count & Type & Description & TPM \\
\hline miR-100-5p & $6,253,908$ & Mature & pmi-miR-100-5p & $236,087.52$ \\
\hline miR-100_2 & $1,150,097$ & Mature & $\begin{array}{l}\text { aga-miR-100,ame-miR-100,lgi-miR-100,sko-miR-100,bmo-miR-100,bma-miR-100b, aae-miR-100,cqu-miR-100-5p,nvi-miR- } \\
\text { 100,mse-miR-100,bbe-miR-100-5p }\end{array}$ & $43,416.62$ \\
\hline miR-184-3p_2 & $1,067,091$ & Mature & $\begin{array}{l}\text { dme-miR-184-3p,dps-miR-184,ame-miR-184,cin-miR-184,tca-miR-184-3p,dan-miR-184-3p,der-miR-184-3p,dgr-miR-184- } \\
\text { 3p,dmo-miR-184-3p,dpe-miR-184-3p,dse-miR-184-3p,dsi-miR-184-3p,dvi-miR-184-3p,dwi-miR-184-3p,dya-miR-184-3p,cte- } \\
\text { miR-184a,lgi-miR-184,sko-miR-184-3p,spu-miR-184,isc-miR-184,api-miR-184a,aae-miR-184,cqu-miR-184,nvi-miR-184,ngi- } \\
\text { miR-184,nlo-miR-184,tur-miR-184-3p,mse-miR-184,hme-miR-184,pmi-miR-184-3p,lva-miR-184-3p,bbe-miR-184-3p }\end{array}$ & $40,283.11$ \\
\hline let-7-5p_5 & 905,569 & Mature & $\begin{array}{l}\text { cel-let-7-5p,cbr-let-7,bfl-let-7a-5p,lgi-let-7,sko-let-7,bfl-let-7a-5p,crm-let-7,ppc-let-7,bma-let-7, asu-let-7-5p,cbn-let-7,bbe-let- } \\
\text { 7a-5p,bbe-let-7a-5p }\end{array}$ & $34,185.59$ \\
\hline miR-2a-3p_5 & 855,173 & Mature & $\begin{array}{l}\text { dme-miR-2a-3p,dme-miR-2a-3p,dps-miR-2a,dps-miR-2a,ame-miR-2,ame-miR-2,aga-miR-2,aga-miR-2,ame-miR-2,bmo-miR- } \\
\text { 2a-3p,bmo-miR-2a-3p,tca-miR-2c-3p,tca-miR-2a-3p,tca-miR-2b-3p,dan-miR-2a,dan-miR-2a,der-miR-2a,der-miR-2a,dgr-miR- } \\
\text { 2a,dgr-miR-2a,dmo-miR-2a,dmo-miR-2a,dpe-miR-2a,dpe-miR-2a,dse-miR-2a,dse-miR-2a,dse-miR-2a,dsi-miR-2a,dsi-miR- } \\
\text { 2a,dvi-miR-2a-3p,dvi-miR-2a-3p,dwi-miR-2a,dwi-miR-2a,dya-miR-2a,dya-miR-2a,dpu-miR-2,dpu-miR-2,isc-miR-2a,aae-miR- } \\
\text { 2c,nvi-miR-2c,ngi-miR-2,nlo-miR-2,hme-miR-2a,hme-miR-2b }\end{array}$ & $32,283.12$ \\
\hline miR-2d_2 & 822,214 & Mature & lgi-miR-2d & $31,038.91$ \\
\hline miR-8-3p_1 & 621,912 & Mature & $\begin{array}{l}\text { dme-miR-8-3p,dps-miR-8, ame-miR-8,aga-miR-8, bmo-miR-8-3p,tca-miR-8-3p,dan-miR-8,der-miR-8, dgr-miR-8, dmo-miR- } \\
\text { 8,dpe-miR-8,dse-miR-8,dsi-miR-8,dvi-miR-8-3p,dwi-miR-8,dya-miR-8,cte-miR-8,lgi-miR-8,dpu-miR-8,isc-miR-8, aae-miR-8- } \\
\text { 3p,nvi-miR-8,nlo-miR-8,mse-miR-8 }\end{array}$ & $23,477.43$ \\
\hline miR-34_5 & 618,791 & Mature & cte-miR-34,bma-miR-34,asu-miR-34-5p,mse-miR-34 & $23,359.61$ \\
\hline miR-375_1 & 557,877 & Mature & lgi-miR-375 & $21,060.08$ \\
\hline miR-1994a & 532,394 & Mature & lgi-miR-1994a & $20,098.09$ \\
\hline miR-2b_8 & 508,766 & Mature & aae-miR-2b & $19,206.12$ \\
\hline miR-279 & 502,037 & Mature & cte-miR-279,api-miR-279a & $18,952.1$ \\
\hline miR-133-3p_4 & 429,969 & Mature & $\begin{array}{l}\text { dme-miR-133-3p,dps-miR-133,ame-miR-133,aga-miR-133,bmo-miR-133,tca-miR-133-3p,dan-miR-133,der-miR-133,dgr- } \\
\text { miR-133,dmo-miR-133,dpe-miR-133,dse-miR-133,dsi-miR-133,dvi-miR-133-3p,dwi-miR-133,dya-miR-133,cte-miR-133,lgi- } \\
\text { miR-133-3p,sko-miR-133,dpu-miR-133,isc-miR-133, aae-miR-133,cqu-miR-133,nvi-miR-133,ngi-miR-133,nlo-miR-133,tur- } \\
\text { miR-133-3p,mse-miR-133,bbe-miR-133-5p }\end{array}$ & $16,231.5$ \\
\hline miR-96a & 391,853 & Mature & lgi-miR-96a & $14,792.61$ \\
\hline miR-92_2 & 384,773 & Mature & dpu-miR-92 & $14,525.33$ \\
\hline miR-1985 & 379,808 & Mature & hru-miR-1985 & $14,337.9$ \\
\hline miR-72-5p & 351,843 & Mature & cel-miR-72-5p,bma-miR-72,asu-miR-72-5p,hco-miR-72,str-miR-72-5p & $13,282.21$ \\
\hline miR-92a_10 & 327,433 & Mature & bfl-miR-92a,bfl-miR-92a,bfl-miR-92a,bbe-miR-92a-3p,bbe-miR-92a-3p & $12,360.73$ \\
\hline miR-184_1 & 302,852 & Mature & aga-miR-184,bfl-miR-184-3p & $11,432.78$ \\
\hline $\operatorname{miR}-315 a$ & 289,278 & Mature & dvi-miR-315a,cte-miR-315,lgi-miR-315,api-miR-315 & $10,920.36$ \\
\hline miR-29a_1 & 279,486 & Mature & cte-miR-29a,lgi-miR-29 & $10,550.71$ \\
\hline miR-750_1 & 273,717 & Mature & cte-miR-750,isc-miR-750 & $10,332.93$ \\
\hline miR-1-3p_4 & 196,893 & Mature & bfl-miR-1-3p,lgi-miR-1,spu-miR-1,pmi-miR-1-3p,lva-miR-1-3p,bbe-miR-1-5p & $7,432.79$ \\
\hline let-7-5p_3 & 147,933 & Mature & $\begin{array}{l}\text { dme-let-7-5p,dps-let-7, bmo-let-7-5p, ame-let-7, dan-let-7, der-let-7,dgr-let-7, dgr-let-7, dmo-let-7,dpe-let-7, dpe-let-7, dse-let- } \\
\text { 7,dsi-let-7,dvi-let-7,dwi-let-7,dya-let-7,cte-let-7,isc-let-7,nvi-let-7,ngi-let-7 }\end{array}$ & $5,584.53$ \\
\hline miR-71c-5p & 134,711 & Mature & sme-miR-71c-5p,tca-miR-71-5p,bfl-miR-71-5p,pmi-miR-71-5p,bbe-miR-71-5p & $5,085.39$ \\
\hline miR-71_5 & 118,698 & Mature & cte-miR-71,dpu-miR-71,isc-miR-71, api-miR-71,tur-miR-71-5p,tur-miR-71-5p,mse-miR-71 & $4,480.9$ \\
\hline miR-87b_2 & 102,530 & Mature & cte-miR-87b,lgi-miR-87 & $3,870.55$ \\
\hline
\end{tabular}

et al., 2013). This technology has been widely utilized to identify conserved and novel miRNAs in various species (Chen et al., 2009, 2012; Ge et al., 2013; Hackl et al., 2011; Lv et al., 2012; Song et al., 2010). In the current study, 104 conserved mg-miRNAs and 33 novel miRNAs were identified by deep sequencing techniques. Some miRNAs revealed to have key roles in multiple biological processes in marine species were also identified in M. galloprovincialis. For example, miR-31 is a conserved and critical regulator of gene expression in many pathogenic processes in vertebrates. It modulates respiratory bursts via targeting Ajp105 during pathological development of the sea cucumber, Apostichopus japonicas (Lu et al., 2015). In the present study, two members of the miR-31 family (miR-31_2, miR-31_3) were identified with high expression levels in M. galloprovincialis. In addition, miR-92a is a disease related regulator that is involved not only in tumorigenesis, but also in multiple host-pathogen interactions in vertebrates. Exposure to bacteria (Vibrio splendidus) and lipopolysaccharides (LPS) significantly increased the expression of A. japonicus miR-92a at different time points (Zhang et al., 2014). In marine shrimp (Marsupenaeus japonicus), miR-7 was upregulated in response to white spot syndrome virus (WSSV) infection and might have an evolutionarily conserved role (Huang and Zhang, 2012). In the current study, both the miR-92a and miR-7 families (miR-7, miR-7-5p_3, and miR-7-5p_5) were expressed at a high level, suggesting that these miRNAs participate in many disease defense pathways in mussels.

Among the conserved miRNAs, five miRNAs (let-7, miR-100, miR184, miR-279, and miR-34) were present in $>10$ million copies in the mg-miRNA libraries. Studies have shown that both the let-7 and miR100 families are highly conserved in different sequences and functions (Christodoulou et al., 2010; Roush and Slack, 2008). The sequences of the mature let-7 families are $100 \%$ conserved among bilaterians (Caygill and Johnston, 2008; Pasquinelli et al., 2000). Temporal regulation of let-7 is also conserved, and its expression is largely limited to developmental stages corresponding to the transitional period between the juvenile and adult individuals in multiple species, such as nematode (Caenorhabditis elegans), fly (Drosophila melanogaster), zebrafish, and rat (Schulman et al., 2005). Drosophila let-7 co-expresses with other miRNA families in numerous tissues late in the third larval instar (L3), peaking in pupae during metamorphosis (Hutvágner et al., 2001; Sempere et al., 
Table 2

The information of potential novel miRNAs in the mussel Mytilus galloprovincialis.

\begin{tabular}{|c|c|c|c|}
\hline miRNA id & Sequence (mature) & Chromosome & Tag information \\
\hline novel_mir1 & uucguugucgucgaaaccugccu & Bpl_scaf_25767 & $\begin{array}{l}\text { tag25633_115,tag22972_1207,tag15046_595921,tag23312_182,- } \\
\text { tag13505_230,tag7249_13753,tag22276_40,tag1874_4208,-- } \\
\text { tag25901_40,tag12914_1720 }\end{array}$ \\
\hline novel_mir2 & uuaaguacuagugccgcgggaa & Bpl_scaf_44438 & $\begin{array}{l}\text { tag13189_208,tag19664_15,tag7216_555,tag26570_902,-- } \\
\text { tag20145_48,tag18681_755,tag18987_10717,tag13053_2140,- } \\
\text { tag9179_10,tag9469_251,tag1908_28,tag24336_51,-- } \\
\text { tag19091_355,tag14944_323064,tag1665_26,tag8217_242671 }\end{array}$ \\
\hline novel_mir3 & ucacaaccuggaugaaugagggc & Bpl_scaf_41094 & tag18740_2356,tag16776_35 \\
\hline novel_mir4 & ucacaaccuccuugaaugagcuu & Bpl_scaf_30185 & $\begin{array}{l}\text { tag22836_1296,tag18995_1416,tag22244_141,tag17627_158,-- } \\
\text { tag22622_159,tag24882_19981,tag181_12,-- } \\
\text { tag16333_200,tag22889_983 }\end{array}$ \\
\hline novel_mir5 & ucccugagaccauaacuugug & Bpl_scaf_35208 & $\begin{array}{l}\text { tag14889_157,tag3628_3549,tag15779_39,tag21630_42564,- } \\
\text { tag14632_467,tag20698_223,tag7309_4349,tag11881_10,- } \\
\text { tag26151_5626,tag18270_153553,tag18411_13,tag3379_808,-- } \\
\text { tag7998_27,tag22016_378,tag4647_1091,tag18720_461,-- } \\
\text { tag9356_38,tag4566_19 }\end{array}$ \\
\hline novel_mir6 & uuacccuguagaaccgagcgagug & Bpl_scaf_61139 & $\begin{array}{l}\text { tag21279_2578,tag7558_33,tag24635_208,tag11394_229,- } \\
\text { tag22378_103137,tag25711_39,tag23727_10,tag20385_122,- } \\
\text { tag14486_96009,tag24517_1741,tag4100_1871,tag3348_27,- } \\
\text { tag17170_29,tag1863_167,tag25109_198,tag24334_37,-- } \\
\text { tag17877_12035,tag24466_64,tag8607_25,tag16303_59 }\end{array}$ \\
\hline novel_mir7 & caaguuacuagccgagauuacg & Bpl_scaf_8330 & $\begin{array}{l}\text { tag6859_11,tag13521_2429,tag4357_23,tag19936_12,-- } \\
\text { tag5685_151,tag21218_1493,tag9702_203,tag21631_187,- } \\
\text { tag6240_5238,tag21551_551,tag12694_30615,tag8232_364 }\end{array}$ \\
\hline novel_mir8 & auuuggcacuuguggaauaauca & Bpl_scaf_19931 & $\begin{array}{l}\text { tag8626_959,tag5186_1145,tag14323_282,tag1066_118,- } \\
\text { tag176_25,tag6793_43314,tag12941_121,tag6981_2143,- } \\
\text { tag6006_28,tag18209_4018,tag10174_1054,tag24844_61210,- } \\
\text { tag23575_23,tag23942_723,tag12661_10 }\end{array}$ \\
\hline novel_mir9 & gagcugccaaaugaagggcug & Bpl_scaf_54119 & $\begin{array}{l}\text { tag4580_57941,tag2205_22,tag5749_112,tag13183_430,- } \\
\text { tag20122_719,tag1321_70 }\end{array}$ \\
\hline novel_mir10 & ugguggucagugcagcgguaga & Bpl_scaf_28009 & $\begin{array}{l}\text { tag25418_131,tag20867_25,tag19867_3733,tag11171_105,- } \\
\text { tag14097_20685,tag7453_131 }\end{array}$ \\
\hline novel_mir11 & ugagaucauugugaaaacugau & Bpl_scaf_40595 & $\begin{array}{l}\text { tag7359_89,tag5746_12796,tag13588_30944,tag26627_71,-- } \\
\text { tag20347_3288,tag4972_666 }\end{array}$ \\
\hline novel_mir12 & ucaguguuguaguaguguuaca & Bpl_scaf_5555 & tag15525_623,tag18094_45,tag13249_13557,tag22837_16 \\
\hline novel_mir13 & uguaccagaucagaguuguua & Bpl_scaf_11649 & $\begin{array}{l}\text { tag1794_4565,tag6598_5625,tag5542_1317,tag13190_64,-- } \\
\text { tag11935_100,tag8997_18,tag15337_617,tag22531_10 }\end{array}$ \\
\hline novel_mir14 & uaaaugcauaaucugguaugug & Bpl_scaf_681 & tag17390_214,tag20463_15,tag6174_3289 \\
\hline novel_mir15 & ucgcugagacaaagaauucuac & Bpl_scaf_63585 & $\begin{array}{l}\text { tag14979_81,tag5247_790,tag13625_12,tag13222_523,- } \\
\text { tag16258_4048,tag7667_61 }\end{array}$ \\
\hline novel_mir16 & uggcgecguguaaacaucucuc & Bpl_scaf_2982 & tag9984_17,tag16275_219,tag16367_1211,tag23335_37 \\
\hline novel_mir17 & gcuuaacacgcggaagguccug & Bpl_scaf_23 & tag22643_229,tag1046_49,tag19822_28 \\
\hline novel_mir18 & gcuuaacacgcggaagguccug & Bpl_scaf_23 & \\
\hline novel_mir19 & gcuuaacacgcggaagguccug & Bpl_scaf_23 & \\
\hline novel_mir20 & ucaccggguauacauucauccg & Bpl_scaf_33037 & tag5135_128,tag2156_367,tag4492_29 \\
\hline novel_mir21 & uaucacagccugcuuugaug & Bpl_scaf_13745 & tag4035_110,tag19513_752,tag18346_5511 \\
\hline novel_mir22 & aauugcaccugucccggccug & Bpl_scaf_50342 & tag10019_399,tag25408_6958,tag1747_39021,tag10138_152 \\
\hline novel_mir23 & uugcguagguguugugcacagu & Bpl_scaf_52347 & tag1824_709 \\
\hline novel_mir24 & cuuggcacuuguagaauucacuga & Bpl_scaf_56410 & $\begin{array}{l}\text { tag11477_74239,tag15593_615,tag10519_72,tag25619_78,- } \\
\text { tag17923_82,tag24934_64,tag4982_88591,tag13186_37,-- } \\
\text { tag19748_111,tag9718_30,tag19599_62,tag24864_27,-- } \\
\text { tag17273_922,tag24944_154,tag26519_15,tag7598_332563,- } \\
\text { tag4265_50866,tag24858_409,tag16933_66,tag4366_81464,- } \\
\text { tag15970_144,tag18847_5238,tag14693_226 }\end{array}$ \\
\hline novel_mir25 & uaucacagccagcuuugaugaca & Bpl_scaf_13745 & $\begin{array}{l}\text { tag1050_37,tag3707_701,tag19490_44,tag10785_3151,-- } \\
\text { tag25427_273,tag12234_66,tag24365_246,tag19305_413,- } \\
\text { tag24621_100,tag25270_44,tag7708_75 }\end{array}$ \\
\hline novel_mir26 & uuuggcacauuuuguaugcuggc & Bpl_scaf_46488 & tag2371_101,tag11108_5672,tag5387_145,tag1236_783 \\
\hline novel_mir27 & cuuggcacuggcggaaugaucac & Bpl_scaf_63435 & $\begin{array}{l}\text { tag7457_32061,tag960_6290,tag9288_18,tag9830_42,- } \\
\text { tag17233_93,tag15253_655,tag20940_14,-- } \\
\text { tag20164_10,tag14534_228 }\end{array}$ \\
\hline novel_mir28 & uaucacagucugcuaugaugagc & Bpl_scaf_33596 & $\begin{array}{l}\text { tag25146_60,tag16247_11,tag22245_14,tag11580_12,-- } \\
\text { tag9676_7403,tag8487_43,tag12098_1300,tag21969_428694,-- } \\
\text { tag5487_539,tag23694_639 }\end{array}$ \\
\hline novel_mir29 & uuuguucguuuggcucgcguu & Bpl_scaf_27638 & $\begin{array}{l}\text { tag9666_631,tag10330_61,tag19238_4902,tag1968_380,- } \\
\text { tag17120_57,tag11757_67591 }\end{array}$ \\
\hline novel_mir30 & ugagacaguguguccucccuaa & Bpl_scaf_46577 & $\begin{array}{l}\text { tag8551_161,tag6326_31,tag11739_139,tag23933_10,- } \\
\text { tag1049_53215,tag12305_433 }\end{array}$ \\
\hline novel_mir31 & cauagcgagaauggccgaguagu & Bpl_scaf_45214 & tag26101_16 \\
\hline novel_mir32 & uuucggacauuauuccugauag & Bpl_scaf_22813 & tag2660_912,tag18802_57,tag23743_13 \\
\hline novel_mir33 & aggcgagccuaaacgauuccgc & Bpl_scaf_23153 & $\begin{array}{l}\text { tag18913_138315,tag15267_50,tag18750_85,tag2498_11,-- } \\
\text { tag20386_62,tag25657_725,tag24463_17159,tag10364_114,-- } \\
\text { tag9150_154,tag24468_950 }\end{array}$ \\
\hline
\end{tabular}




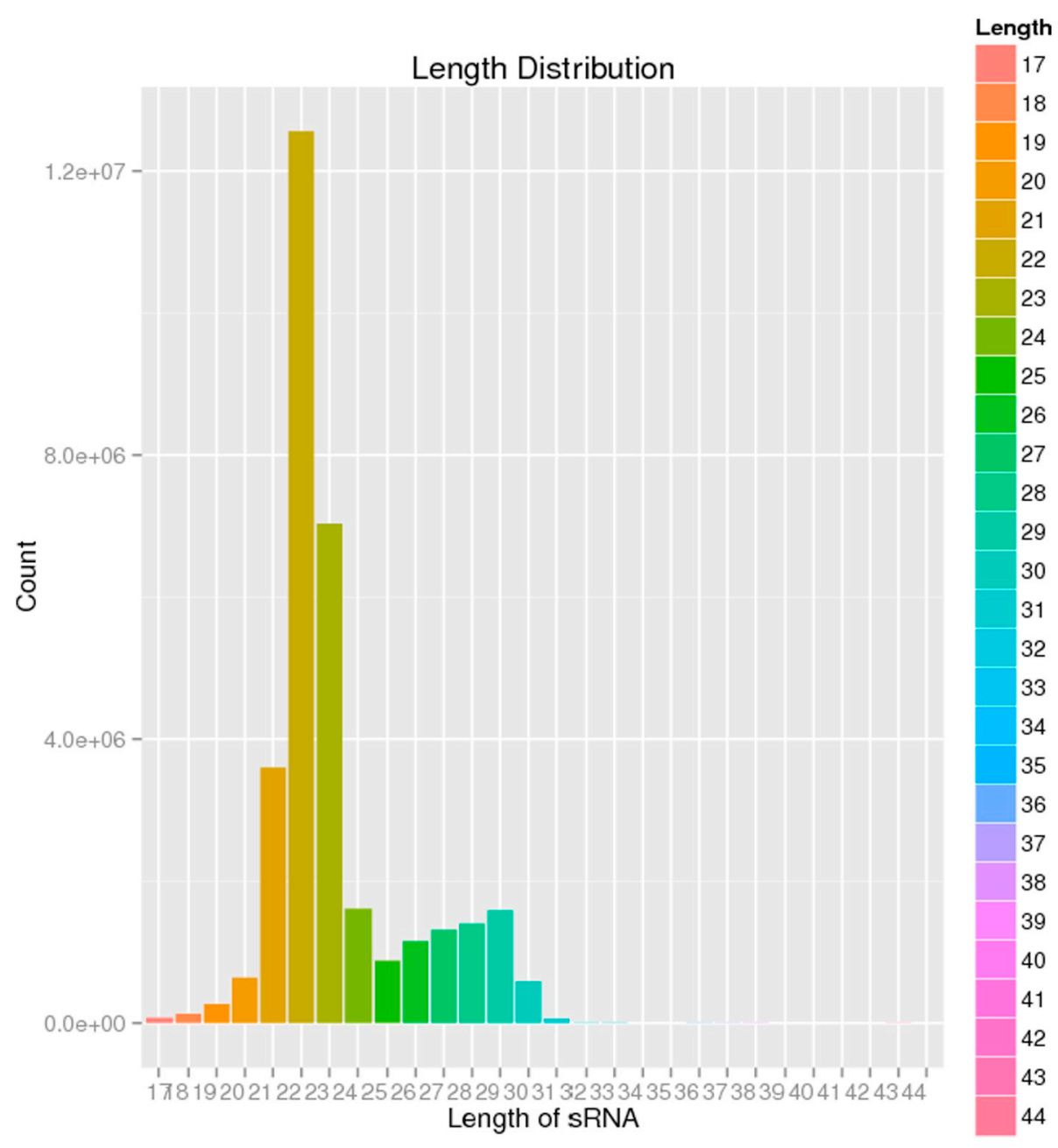

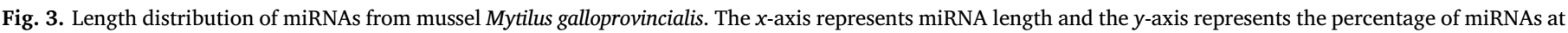
each length. The length distribution analysis provides information that helps further understanding of the compositions of the miRNA samples.

2002). MiR-100 s are considered to be ancient and conserved because they are observed in most metazoans. In the current study, four members of the miR-100 family (miR-100_2, miR-100-5p, miR-100a-5p, and miR-100-5p_4) were identified in M. galloprovincialis, based on conserved miRNA expression patterns (e.g. miR-100, miR-31, miR-34, miR92, and miR-124) (Grimson et al., 2008; Lee et al., 2016; Martín-Gómez et al., 2014b; Mulder and Berezikov, 2010). Early studies demonstrated a central role for the miR-34 family in promoting cell cycle arrest and cell death following stress in human cells. However, the biological significance of this response is unclear in M. galloprovincialis.

Interestingly, a total of 33 novel mg-miRNAs were identified (Table 2) and further revealed to be $M$. galloprovincialis specific. The read numbers of each novel miRNA were lower than those of the majority of the conserved miRNAs. These results are consistent with previous reports stating that nonconserved miRNAs are usually expressed at lower levels than conserved miRNAs (Liu et al., 2010; Zhu et al., 2012). Such a finding might indicate development-specific functions of these novel miRNAs in specific species. However, further studies could be performed to determine whether scarce miRNAs are expressed at higher levels in different developmental stages. Future functional studies will also provide insights into the functions of novel miRNAs in $M$. galloprovincialis.

In this study, miRNAs and mRNAs from the soft tissues of $M$. galloprovincialis were sequenced using the BGISEQ-500 sequencing platform. A total of 104 known miRNAs were identified and 33 potential novel miRNAs were predicted. In addition, both GO enrichment and KEGG pathway analyses of target genes from the three miRNA libraries were carried out. The results of KEGG pathway analyses revealed several enriched terms involved in $M$. galloprovincialis soft tissues (e.g., metabolic pathways, focal adhesion, PI3K-Akt signaling pathway, endocytosis, and neuroactive ligand-receptor interactions), which might affect the balance of the internal environment. Thus, future studies should be performed to determine whether miRNAs and mRNAs are involved in the regulation of environmental adaptation in M. galloprovincialis.

\section{Conclusion}

This study provides the first indication of miRNA and mRNA expression in the soft tissues of $M$. galloprovincialis. These results will be helpful for furthering the study of miRNA regulation and function in $M$. galloprovincialis.

\section{Declaration of competing interest}

The authors declare no conflict of interest. 


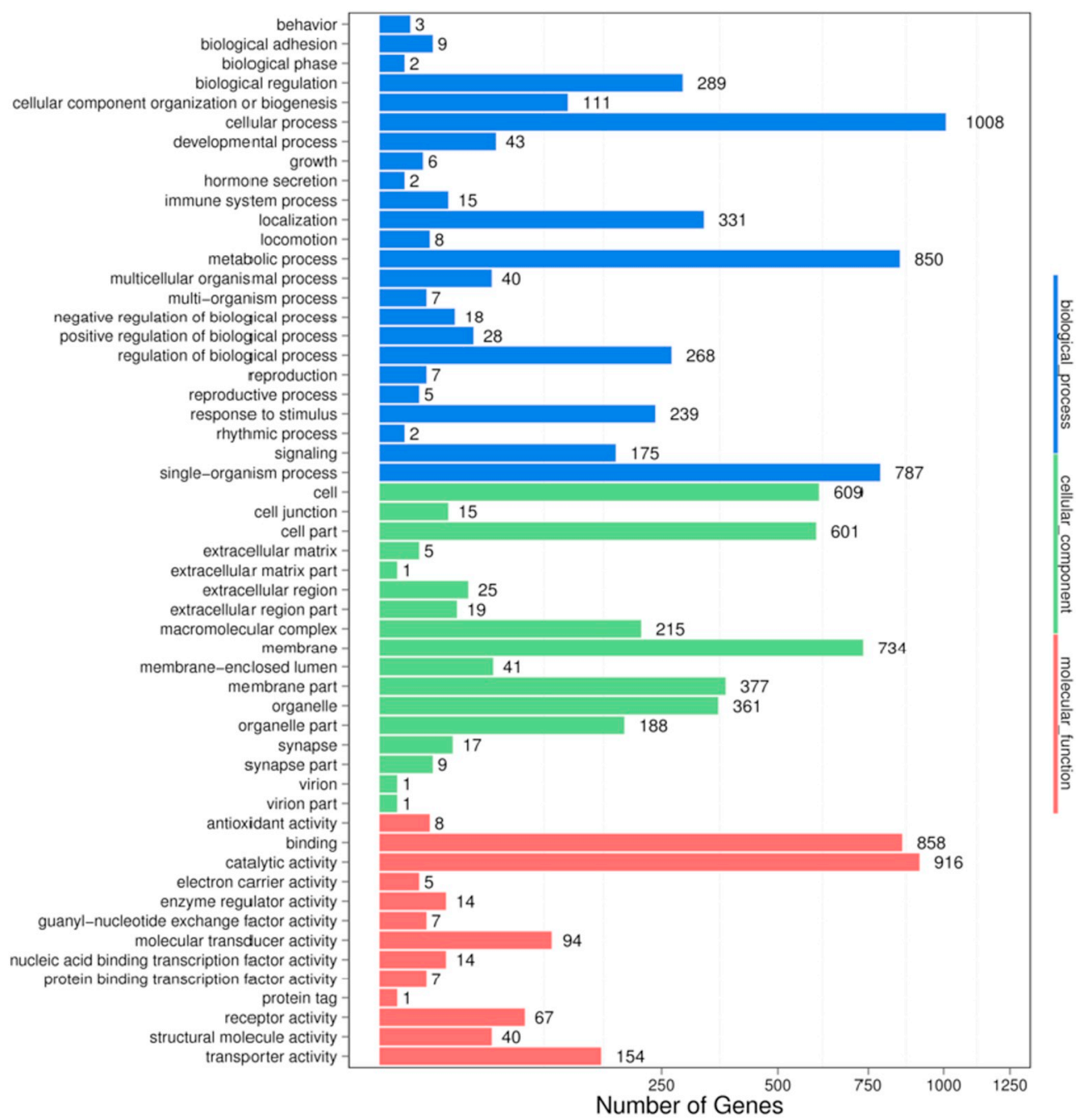

Fig. 4. GO functional classification of mussel Mytilus galloprovincialis. The $x$-axis indicates the number of DEGs (presented by its square root value) and the $y$-axis represents GO terms. All GO terms were grouped into three ontologies: blue indicates biological process, brown indicates cellular component, and orange indicates molecular function. (For interpretation of the references to color in this figure legend, the reader is referred to the web version of this article.)

\section{Acknowledgments}

This work has been partially supported by the National Key Research and Development Program of China (2018YFC0910602); the National Natural Science Foundation of China (31771584/41806142// 61525503/61620106016/61835009/41976152); (Key) Project of Department of Education of Guangdong Province (2016KCXTD007); Guangdong Natural Science Foundation Innovation Team (2014A030312008); Shenzhen Basic Research Project (JCYJ20170818100153423/JCYJ20170412105003520); Natural Science Foundation of SZU (No. 2017000193); China Postdoctoral Science Foundation (No. 2018M643151); and Shandong Key Laboratory of Coastal Environmental Processes, YICCAS (No. 2019SDHADKFJJ03).

\section{References}

Aboobaker, A.A., Tomancak, P., Patel, N., Rubin, G.M., Lai, E.C., 2005. Drosophila microRNAs exhibit diverse spatial expression patterns during embryonic development. Proc. Natl. Acad. Sci. 102 (50), 18017-18022.

Alimba, C., Faggio, C., 2019. Microplastics in the marine environment: current trends in environmental pollution and mechanisms of toxicological profile. Science of the Total Environmental 68, 61-74.

Ambros, V., 2001. microRNAs: tiny regulators with great potential. Cell 107 (7), 823-826. Ambros, V., 2004. The functions of animal microRNAs. Nature 431 (7006), 350-355.

Bartel, D.P., 2004. MicroRNAs: genomics, biogenesis, mechanism. and function. Cell 116
(2), 281-297.

Bartel, D.P., 2018. Metazoan microRNAs. Cell 173 (1), 20-51.

Bueno, M.J., Malumbres, M., 2011. MicroRNAs and the cell cycle. Biochimica et Biophysica Acta-molecular Basis of Disease 1812, 592-601.

Burgos-Aceves, M.A., Cohen, A., Paolella, G., Lepretti, M., Smith, Y., Faggio, C., Lionetti, L., 2018a. Modulation of mitochondrial functions by xenobiotic-induced microRNA: from environmental sentinel organisms to mammals. Sci. Total Environ. 645, 79-88.

Burgos-Aceves, M.A., Cohen, A., Smith, Y., Faggio, C., 2018b. MicroRNAs and their role on fish oxidative stress during xenobiotic environmental exposures. Ecotoxicol. Environ. Saf. 148, 995-1000.

Burgos-Aceves, M.A., Cohen, A., Smith, Y., Faggio, C., 2018c. A potential microRNA regulation of immune-related genes in invertebrate haemocytes. Sci. Total Environ. 621, 302-307.

Capillo, G., Silvestro, S., Sanfilippo, M., Fiorino, E., Giangrosso, G., Ferrantelli, V., Vazzana, I., Faggio, C., 2018. Assessment of electrolytes and metals profile of the Faro Lake (Capo Peloro Lagoon, Sicily, Italy) and its impact on Mytilus galloprovincialis. Chem. Biodivers. 15.

Caygill, E.E., Johnston, L.A., 2008. Temporal regulation of metamorphic processes in Drosophila by the let-7 and miR-125 heterochronic microRNAs. Curr. Biol. 18 (13), 943-950.

Chen, X., Li, Q.B., Wang, J., Guo, X., Jiang, X.R., Ren, Z.J., Weng, C.Y., Sun, G.X., Wang, X.Q., Liu, Y.P., Ma, L.J., Chen, J.Y., Wang, J., Zen, K., Zhang, J.F., Zhang, C.Y., 2009. Identification and characterization of novel amphioxus microRNAs by Solexa sequencing. Genome Biol. 10 (7), R78.

Chen, C., Deng, B., Qiao, M., Zheng, R., Chai, J., Ding, Y., Peng, J., Jiang, S.W., 2012 Solexa sequencing identification of conserved and novel microRNAs in backfat of large white and Chinese Meishan pigs. PLoS One 7 (2), e31426.

Chen, G.F., Zhang, C.Y., Jiang, F.J., Wang, Y.Y., Xu, Z., Wang, C.M., 2014. Bioinformatics analysis of hemocyte miRNAs of scallop Chlamys farreri against acute viral necrobiotic virus (AVNV). Fish Shellfish Immunol. 37 (1), 75-86.

Chen, H., Zhou, Z., Wang, L.L., Wang, H., Liu, R., Zhang, H., Song, L.S., 2016. An invertebrate-specific miRNA targeted the ancient cholinergic neuroendocrine system of 
oyster. Open Biol. 6 (8), 160059.

Chen, H., Xin, L.S., Song, X.R., Wang, L., Wang, W.L., Liu, Z.Q., Zhang, H., Wang, L.L., Zhou, Z., Qiu, L.M., Song, L.S., 2017. A norepinephrine-responsive miRNA directly promotes CgHSP90AA1 expression in oyster haemocytes during desiccation. Fish Shellfish Immunol. 64, 297-307.

Christodoulou, F., Raible, F., Tomer, R., Simakov, O., Trachana, K., Klaus, S., Snyman, H., Hannon, G.J., Bork, P., Arendt, D., 2010. Ancient animal microRNAs and the evolution of tissue identity. Nature 463 (7284), 1084-1088.

Faggio, C., Pagano, M., Alampi, R., Vazzana, I., Felice, M.R., 2016. Cytotoxicity, haemolymphatic parameters, and oxidative stress following exposure to sub-lethal concentrations of quaternium-15 in Mytilus galloprovincialis. Aquat. Toxicol. 180, 258-265.

Faggio, C., Tsarpali, V., Dailianis, S., 2018. Mussel digestive gland as a model for assessing xenobiotics: an overview. Sci. Total Environ. 613, 220-229.

Farrington, J., Tripp, B., Tanabe, S., Subramanian, A., Sericano, J., Wade, T., Knap, A., 2016. Edward D. Goldberg's proposal of "the mussel watch": reflections after 40 years. Mar. Pollut. Bull. 110 (1), 501-510.

Freitas, R., Silvestro, S., Coppola, F., Meucci, V., Battaglia, F., Intorre, L., Soares, A.M.V.M., Pretti, C., Faggio, C., 2019. Biochemical and physiological responses induced in Mytilus galloprovincialis after a chronic exposure to salicylic acid. Aquat. Toxicol. 214, 105258

Ge, X., Zhang, Y., Jiang, J.H., Zhong, Y., Yang, X.N., Li, Z.Q., Huang, Y.P., Tan, A.J., 2013. Identification of MicroRNAs in Helicoverpa armigera and Spodoptera litura based on deep sequencing and homology analysis. Int. J. Biol. Sci. 9 (1), 1-15.

Grimson, A., Srivastava, M., Fahey, B., Woodcroft, B.J., Chiang, H.R., King, N., Degnan, B.M., Rokhsar, D.S., Bartel, D.P., 2008. Early origins and evolution of microRNAs and piwi-interacting RNAs in animals. Nature 455 (7217), 1193-1197.

Hackl, M., Jakobi, T., Blom, J., Doppmeier, D., Brinkrolf, K., Szczepanowski, R., Bernhart, S.H., Siederdissend, C.H.Z., Bort, J.A.H., Wieser, M., Kunert, R., Jeffs, S., Hofacker, I.L., Goesmann, A., Puhler, A., Borth, N., Grillari, J., 2011. Next-generation sequencing of the Chinese hamster ovary microRNA transcriptome: identification, annotation and profiling of microRNAs as targets for cellular engineering. J. Biotechnol. 153 (1-2), 62-75.

He, Y.D., Sun, Y.C., Zhang, X.B., 2017. Noncoding miRNAs bridge virus infection and host autophagy in shrimp in vivo. FASEB J. 31, 2854-2868.

Hoen, P.A.C., Ariyurek, Y., Thygesen, H.H., Vreugdenhil, E., Vossen, R.H., Menezes, R.X., Boer, J.M., Ommen, G.B., Dunnen, J.T., 2008. Deep sequencing-based expression analysis shows major advances in robustness, resolution and inter-lab portability over five microarray platforms. Nucleic Acids Res. 36 (21), e141.

Huang, T.Z., Zhang, X.B., 2012. Functional analysis of a crustacean microRNA in hostvirus interaction. J. Virol. 86 (23), 12997-13004.

Huang, X.D., Zhao, M., Liu, W.G., Guan, Y.Y., Shi, Y., Wang, Q., Wu, S.Z., He, M.X., 2013. Gigabase-scale transcriptome analysis on four species of pearl oysters. Mar. Biotechnol. 15 (3), 253-264.

Huo, D., Sun, L.N., Li, X.N., Ru, X.S., Liu, S.L., Zhang, L.B., Xing, L.L., Yang, H.S., 2017. Differential expression of miRNAs in the respiratory tree of the sea cucumber, Apostichopus japonicus, under hypoxia stress. G3-Genes Genomes Genetics 7 (11), 3681-3692.

Hutvágner, G., Mclachlan, J., Pasquinelli, A.E., Bálint, É., Tuschl, T., Zamore, P.D., 2001. A cellular function for the RNA-interference enzyme dicer in the maturation of the let-7 small temporal RNA. Science 293 (5531), 834-838.

Ji, C.L., Wu, H.F., Wei, L., Yu, J.B., 2013. Proteomic and metabolomic analysis reveal gender-specific responses of mussel Mytilus galloprovincialis to 2,2',4,4'-tetrabromodiphenyl ether (BDE 47). Aquat. Toxicol. 140-141, 449-457.

Jiao, Y., Zheng, Z., Du, X.D., Wang, Q.H., Huang, R.L., Deng, Y.W., Shi, S.L., Zhao, X.X., 2014. Identification and characterization of MicroRNAs in pearl oyster Pinctada martensii by Solexa deep sequencing. Mar. Biotechnol. 16 (1), 54-62.

Kanehisa, M., Araki, M., Goto, S., Hattori, M., Hirakawa, M., Itoh, M., Katayama, T., Kawashima, S., Okuda, S., Tokimatsu, T., Yamanishi, Y., 2008. KEGG for linking genomes to life and the environment. Nucleic Acids Res. 36 (1), 480-484.

Kenny, N.J., Namigai, E.K.O., Marletaz, F., Hui, J.H.L., Shimeld, S.M., 2015. Draft genome assemblies and predicted microRNA complements of the intertidal lophotrochozoans Patella vulgata (Mollusca, Patellogastropoda) and Spirobranchus (Pomatoceros) lamarcki (Annelida, Serpulida). Mar. Genomics 24, 139-146.

Lai, K.P., Li, J.W., Wang, S.Y., Chiu, J.M., Tse, A., Lau, K., Lok, Si., Au, D.W., Tse, W. K., Wong, C.K., Chan, T., Kong, R.Y., Wu, R.S., 2015. Tissue-specific transcriptome assemblies of the marine medaka Oryzias melastigma and comparative analysis with the freshwater medaka Oryzias latipes. BMC Genomics 16 (1), 135.

Lee, H., Han, S., Kwon, C., Lee, D., 2016. Biogenesis and regulation of the let-7 miRNAs and their functional implications. Protein and Cell 7 (2), 100-113.

Liu, S., Li, D., Li, Q., Zhao, P., Xiang, Z.H., Xia, Q.Y., 2010. MicroRNAs of Bombyx mori identified by Solexa sequencing. BMC Genomics 11 (1), 148.

Lu, M., Zhang, P.J., Li, C.H., Zhang, W.W., Jin, C.H., Han, Q.X., 2015. MiR-31 modulates coelomocytes ROS production via targeting p105 in Vibrio splendidus challenged sea cucumber Apostichopus japonicus in vitro and in vivo. Fish Shellfish Immunol. 45 (2), 293-299.

Lv, S.Z., Nie, X.J., Wang, L., Du, X.H., Biradar, S.S., Jia, X.O., Weining, S., 2012. Identification and characterization of microRNAs from barley (Hordeum vulgare L.) by high-throughput sequencing. Int. J. Mol. Sci. 13 (3), 2973-2984.

Martín-Gómez, L., Villalba, A., Carballal, M.J., Abollo, E., 2014a. Molecular characterisation of TNF, AIF, dermatopontin and VAMP genes of the flat oyster Ostrea edulis and analysis of their modulation by diseases. Gene 533 (1), 208-217.

Martín-Gómez, L., Villalba, A., Kerkhoven, R.H., Abollo, E., 2014b. Role of microRNAs in the immunity process of the flat oyster Ostrea edulis against bonamiosis. Infect. Genet. Evol. 27, 40-50.

Mulder, K.D., Berezikov, E., 2010. Tracing the evolution of tissue identity with microRNAs. Genome Biol. 11 (3), 111.

Pagano, M., Capillo, G., Sanfilippo, M., Palato, S., Trischitta, F., Manganaro, A., Faggio, C., 2016. Evaluation of functionality and biological responses of Mytilus galloprovincialis after exposure to quaternium-15 (methenamine 3-chloroallylochloride). Molecules. 21 (2), E144.

Pagano, M., Porcino, C., Briglia, M., Fiorino, E., Vazzana, M., Silvestro, S., Faggio, C., 2017. The influence of exposure of cadmium chloride and zinc chloride on haemolymph and digestive gland cells from Mytilus galloprovincialis. International Journal of Environmental Research 11 (2), 207-216.

Pasquinelli, A.E., Reinhart, B.J., Slack, F., Martindale, M.Q., Kuroda, M.I., Maller, B., Hayward, D.C., Ball, E.E., Degnan, B., Müller, P., Spring, J., Srinivasan, A., Fishman, M., Finnerty, J., Corbo, J., Levine, M., Leahy, P., Davidson, E., Ruvkun, G., 2000. Conservation of the sequence and temporal expression of let-7 heterochronic regulatory RNA. Nature 408 (6808), 86-89.

Roush, S., Slack, F.J., 2008. The let-7 family of microRNAs. Trends Cell Biol. 18 (10), 505-516.

Schulman, B.R.M., Esquela-Kerscher, A., Slack, F.J., 2005. Reciprocal expression of lin-41 and the microRNAs let-7 and mir-125 during mouse embryogenesis. Dev. Dyn. 234 (4), 1046-1054.

Sempere, L.F., Dubrovsky, E.B., Dubrovskaya, V.A., Berger, E.M., Ambros, V., 2002. The expression of the let-7 small regulatory RNA is controlled by ecdysone during metamorphosis in Drosophila melanogaster. Dev. Biol. 244 (1), 170-179.

Simakov, O., Marletaz, F., Cho, S.J., Edsinger-Gonzales, E., Havlak, P., Hellsten, U., Kuo, D.H., Larsson, T., Lv, J., Arendt, D., Savage, R., Osoegawa, K., de Jong, P., Grimwood, J., Chapman, J.A., Shapiro, H., Aerts, A., Otillar, R.P., Terry, A.Y., Boore, J.L., Grigoriev, I.V., Lindberg, D.R., Seaver, E.C., Weisblat, D.A., Putnam, N.H., Rokhsar, D.S., 2013. Insights into bilaterian evolution from three spiralian genomes. Nature 493 (7433), 526-531.

Song, C.N.A., Wang, C., Zhang, C.Q., Korir, N.K., Yu, H.P., Ma, Z.Q., Fang, J.G., 2010 Deep sequencing discovery of novel and conserved microRNAs in trifoliate orange (Citrus trifoliata). BMC Genomics 11, 431.

Stara, A., Pagano, M., Capillo, G., et al., 2020. Assessing the effects of neonicotinoid insecticide on the bivalve mollusc Mytilus galloprovincialis. Sci. Total Environ. 134914, 700 .

Sun, J., Wang, M., Wang, H., Zhang, H., Zhang, X., Thiyagarajan, V., Qian, P.Y., Qiu, J.W., 2012. De novo assembly of the transcriptome of an invasive snail and its multiple ecological applications. Mol. Ecol. Resour. 12 (6), 1133-1144.

Sun, J., Zhang, Y., Xu, T., Zhang, Y., Mu, H., Zhang, Y.J., Lan, Y., Fields, C.J., Hui, J.H.L., Zhang, W.P., Li, R.S., Nong, W.Y., Cheung, F.K.M., Qiu, J.W., Qian, P.Y., 2017. Adaptation to deep-sea chemosynthetic environments as revealed by mussel genomes. Nat. Ecol. Evol. 1 (5), 121.

Torre, A., Trischitta, F., Corsaro, C., Mallamace, D., Faggio, C., 2013. Digestive cells from Mytilus galloprovincialis show a partial regulatory volume decrease following acute hypotonic stress through mechanisms involving inorganic ions. Cell Biochem. Funct. 31, 489-495.

Yu, D.L., Ji, C.L., Zhao, J.M., Wu, H.F., 2016. Proteomic and metabolomic analysis on the toxicological effects of As (III) and As (V) in juvenile mussel Mytilus galloprovincialis. Chemosphere 150, 194-201.

Zhang, P.J., Li, C.H., Shao, Y.N., Chen, X.C., Li, Y., Su, X.R., Li, T.W., 2014. Identification and characterization of miR-92a and its targets modulating Vibrio splendidus challenged Apostichopus japonicas. Fish Shellfish Immunol. 38 (2), 383-388.

Zheng, Z., Jiao, Y., Du, X.D., Tian, Q.L., Wang, Q.H., Huang, R.L., Deng, Y.W., 2016. Computational prediction of candidate miRNAs and their potential functions in biomineralization in pearl oyster Pinctada martensii. Saudi Journal of Biological Sciences 23 (3), 372-378.

Zhou, Z., Wang, L.L., Song, L.S., Liu, R., Zhang, H., Huang, M.M., Chen, H., 2014. The identification and characteristics of immune-related microRNAs in haemocytes of oyster Crassostrea gigas. PLoS One 9 (2), e88397.

Zhu, Y.P., Xue, W., Wang, J.T., Wan, Y.M., Wang, S.L., Xu, P., Zhang, Y., Li, J.T., Sun, X.W., 2012. Identification of common carp (Cyprinus carpio) microRNAs and microRNA-related SNPs. BMC Genomics 13 (1), 413. 